\title{
Two new scales in the field of couples and marriage counseling: The inclusion of other in the self scale and Turkish self-change in romantic relationships scale
}

\author{
Duygu Dincer ${ }^{1 *}$, Halil Eksi ${ }^{2}$, and Arthur Aron $^{3}$ \\ ${ }^{1}$ Ibn Haldun University, Department of Counseling Psychology, 34494 Istanbul, Turkey \\ ${ }^{2}$ Marmara University, Department of Counseling Psychology, 34722 Istanbul, Turkey \\ ${ }^{3}$ Stony Brook University, Department of Psychology, 11794 New York, USA
}

\begin{abstract}
This study had two aims. One aim was to adapt the Inclusion of Other in the Self (IOS) Scale into the Turkish cultural context. The second aim was to develop the Turkish Self-Change in Romantic Relationships Scale (TSCRRS) based on the existing Relational Self-Change Scale. The research process for this study consisted of four stages. In the first stage, forward-backward translation of the IOS Scale was performed to determine bilingual equivalence. In the second stage, an item-pool was created to measure self-change in romantic relationships. In the third stage, data were collected to determine the reliability and validity of the TSCRRS (N $=426$ ). In the fourth stage, new data were collected to determine the validity and reliability of the IOS Scale and the TSCRRS $(\mathrm{N}=348)$. All of the participants were in a romantic relationship. The findings revealed that both the TSCRRS and the IOS Scale have good reliability and validity.
\end{abstract}

Keywords: romantic relationships, self-change, the inclusion of other in the self, scale adaptation, scale development, culture

\section{Introduction}

People in a romantic relationship can experience positive and negative changes related to their self-concepts. In 2014, three social psychology researchers, Brent Mattingly, Gary Lewandowski, and Kevin McIntyre proposed a new psychological model called the Twodimensional Model of Relational Self-change to conceptualize these self-changes, and they developed a scale based on their model [1]. According to this model, self-changes in a romantic relationship can occur based on two dimensions: direction and valence $[1,2]$. The concept of direction refers to a quantitative self-change and the concept of valence refers to a qualitative self-change. Thus, the direction of the self-concept is related to increases or decreases in the content of an individual's self-concept, but the direction of the self-concept is associated with the subjective aspects of the changes in that person's self-concept $[1,2]$. Based on this conceptualization, there are four types of self-concept changes: selfexpansion, self-pruning, self-contraction, and self-adulteration [1]. These self-concept changes can be formularized as follows:

* Corresponding author: drduygudincer@gmail.com 
- Self-expansion $=$ Positive content of self-concept $\uparrow$

- Self-contraction $=$ Positive content of self-concept $\downarrow$

- Self-adulteration = Negative content of self-concept $\uparrow$

- Self-pruning $=$ Negative content of self-concept $\downarrow$

Although this model introduced and conceptualized the terms of self-pruning, selfcontraction, and self-adulteration for the first time, the concept of self-expansion has been known for many years. Almost three decades ago, two social psychology researchers, Arthur Aron and Elaine Aron, developed the Self-expansion Theory that assumes people have a motive to expand their selves, and one of the best ways of accomplishing that is to establish and maintain close relationships, especially romantic relationships [3, 4]. According to this theory, people seek out and create an optimal close relationship [3], and they tend to include in the self the other's social, intellectual, and financial resources, worldview, and identities [5]; thus, they become a collective unit and begin to see and experience the world from their partner's viewpoint [6].

Although many researchers from all over the world have examined the ideas stated above, there seems to have been little interest in the Turkish close relationships literature on self-changes and the inclusion of other in the self. Thus, the present study aimed to draw attention to these relational dynamics and provide psychological tools to measure these relational constructs. For this reason, we tested the validity and reliability of the Inclusion of Other in the Self (IOS) Scale [7] and we developed a new scale to assess self-change in romantic relationships based on the Two-dimensional Model of Relational Self-change.

\section{Method}

\subsection{Sample and procedures}

In the first stage of this study, forward-backward translation of the IOS Scale [7] was performed to determine bilingual equivalence. Then, 31 students (all currently in a dating relationship) from the English Teaching Department, who were assumed to be fluent in both English and Turkish, participated in a bilingual equivalence study. Twenty-five of the participants $(80.6 \%)$ were female, and six $(19.4 \%)$ were male. The participants ranged in age from 18 to 39 ; the mean was 22.6 years $(D F=3.68)$. The length of their relationships ranged from 5 months to 120 months; the mean relationship length was 28.9 months $(D F=$ 2.47),

In the second stage, based on the existing Relational Self-Change Scale (RSCS) [1], the Two-dimensional Model of the Relational Self-change [1], and a focus group conducted with Turkish college students were used to create an item-pool to measure self-change processes in romantic relationships. Then, these items were examined by four specialists in the field of close relationships. Based on that feedback, the authors created the final itempool $[8,9]$

In the third stage, data were collected to determine the reliability and validity of the Turkish Self-Change in Romantic Relationships Scale (TSCRRS) $(\mathrm{N}=426$, all currently in a romantic relationship). Of the 426 participants in this stage of the study, $210(49.3 \%)$ were female and $216(50.7 \%)$ were male. Moreover, $70.1 \%(\mathrm{~N}=285)$ of the participants were dating, $14.6 \%(\mathrm{~N}=62)$ were engaged, and $18.3 \%(\mathrm{~N}=78)$ were married. The participants' ages ranged from 18 to 40 ; the mean age was 23.88 years $(D F=4.63)$. The length of the participants' relationships ranged from 1 month to 192 months; the mean relationship length was 31.7 months $(D F=31.22)$. In this sample, exploratory factor 
analysis (EFA) was applied to discover the factor structure of the scale and to examine its internal reliability.

In the fourth stage, new data were collected to determine the construct validity (via confirmatory factor analysis [CFA]) and the parallel test reliability of the TSCRRS, and to assess the criterion validity of the IOS Scale and the TSCRRS $(\mathrm{N}=348$, all currently in a romantic relationship). Of the 348 participants in this stage of the study, 141 (40.5\%) were female and $207(59.5 \%)$ were male; moreover, $70.0 \%(\mathrm{~N}=240)$ of the participants were dating, $13.5 \%(\mathrm{~N}=47)$ were engaged, and $17.5 \%(\mathrm{~N}=61)$ were married. The participants ranged in age from 18 to 40 ; the mean age was 23.71 years $(D F=4.60)$. The length of the relationships ranged from 1 month to 144 months; the mean relationship length was 30.3 months $(D F=29.98)$. The existing RSCS [1] was used as a parallel test of the TSCRRS (Actually, the TSCRRS was developed as the parallel test of the RSCS in the first author's dissertation [8]). Furthermore, the Relationship Assessment Scale (RAS) [10] were used to determine the criterion validity of the TSCRRS. At the same time, the RAS and the RSCS were used to determine criterion validity of the IOS Scale.

\subsection{Materials}

In this study, the participants completed the IOS Scale, the Turkish version of the RSCS, the RAS, and the TSCRRS. They also completed a brief demographic information sheet which consisted of some questions about their gender, age, current relationship status, type of relationship, and the duration of the relationship.

\subsubsection{The Inclusion of Other into the Self (IOS) Scale:}

The one-item pictorial IOS Scale was developed to assess the self-other overlap in romantic relationships [7]. The scale consists of seven paired circles. In each pair, one circle represents the self, and one circle represents the other (partner). The degree of intersection indicates the degree of closeness and intimacy, and the extent to which the other is included in the self. In the original study, the scale demonstrated good reliability $(\alpha=.95$; and testretest reliability was .85) and validity (As expected, the IOS Scale was positively related to the Sternberg Intimacy Scale and the Subjective Closeness Index) [7].

\subsubsection{The Relational Self-Change Scale (RSCS):}

The RSCS was developed to assess changes in self-concepts [1]. It consists of four subscales: self-expansion, self-contraction, self-pruning, and self-adulteration. Each subscale contains three items. Sample items include: "I have learned many great new things" (self-expansion), "My positive attributes have decreased" (self-contraction), "My bad habits have diminished" (self-pruning), and "I have more negative qualities" (selfadulteration). The RSCS is rated on a 7 -point scale $(1=$ not very much and $7=$ very much). In the original research, each subscale demonstrated good reliability. In the first study at Time 1 (the first time the RSCS was administered), the following reliability results were obtained: self-expansion $(\alpha=.81)$, self-contraction $(\alpha=.72)$, self-pruning $(\alpha=.78)$, and self-adulteration $(\alpha=.72)$. At Time 2 (the second time the RSCS was administered) the reliability results were: self-expansion $(\alpha=.85)$, self-contraction $(\alpha=.84)$, self-pruning ( $\alpha$ $=.78)$, and self-adulteration $(\alpha=.72)$. In the second study, the reliability results were: selfexpansion $(\alpha=.84)$, self-contraction $(\alpha=.92)$, self-pruning $(\alpha=.92)$, and self-adulteration $(\alpha=.78)$. Consistent with these findings, in another study conducted by the same authors, 
each subscale again demonstrated good reliability. At Time 1, the following reliability results were found: self-expansion $(\alpha=.81)$, self-contraction $(\alpha=.72)$, self-pruning $(\alpha=$ $.78)$, and self-adulteration $(\alpha=.72)$. At Time 2 , the reliability results were: self-expansion $(\alpha=.85)$, self-contraction $(\alpha=.84)$, pruning $(\alpha=.88)$, and adulteration $(\alpha=.79)$. The scale was adapted to the Turkish cultural context by the authors of the current paper $[8,9]$. The findings revealed that the goodness of fit index values were good $\left[x^{2} / D F=2.34, x^{2}=\right.$ 112.18, $\mathrm{DF}=48, \mathrm{CFI}=.98, \mathrm{NFI}=.96, \mathrm{GFI}=.96, \mathrm{RMSEA}=.06(\mathrm{~N}=426)]$. Moreover, Cronbach's alpha coefficients of $.84, .84, .89$, and .76 were obtained for the self-expansion, self-contraction, self-pruning, and self-adulteration subscales. It was shown that the inclusion of other in the self and relationship satisfaction were positively correlated to selfexpansion $(.33, p<.01 ; .42, p<.01)$ and self-pruning $(.27, p<.01 ; .25, p<.01)$, and they were negatively correlated to self-contraction $(-.19, p<.01 ;-.45, p<.01)[1,2]$.

\subsubsection{The Relationship Assessment Scale (RAS):}

The 7-item and single structure RAS was developed to assess global relationship satisfaction [10]. It is rated on a 5-point scale $(1=$ low satisfaction and $7=$ high satisfaction). Sample items include: "How good is your relationship compared to most?" and "To what extent has your relationship met your original expectations?". In the original study, the scale demonstrated good reliability $(\alpha=.86)$ and validity; in fact, the explained variance was high (46\%) [10]. The RAS was adapted to the Turkish cultural context [11], and it was found that the scale had good validity and reliability $(\alpha=.86$; the explained variance was $52 \%$ in the total sample).

\subsubsection{The Turkish Self-Change in Romantic Relationships Scale (TSCRRS):}

The authors of the current paper developed the 16-item TSCRRS based on the existing Relational Self-Change Scale (RSCS) [1], the Two-dimensional Model of the Relational Self-change [1], and a focus group conducted with Turkish college students to assess the perceived self-change processes in couple relationships. The TSCRRS consists of four subscales: self-expansion (5 items), self-contraction (5 items), self-pruning (3 items), and self-adulteration ( 3 items). The TSCRRS is rated on a 7-point scale $(1=$ not very much and 7 = very much). Moreover, it contains several culture-specific items based on a focus group.

\section{Results}

\subsection{The validity of the TSCRRS}

\subsubsection{EFA}

The Kaiser-Meyer-Olkin (KMO) score for the TSCRRS was 0.89, and Bartlett's Test of Sphericity $\left(\chi^{2}=4113.31, D F=231, p<.00\right)$ was found to be significant. Based on the $\mathrm{KMO}$ and Bartlett's Test of Sphericity scores, factor analysis was conducted. EFA was performed to evaluate the TSCRRS. The EFA results support the use of a 16-item fourfactor solution as the best fitting model for the data, and the explained variance was high $(62.34 \%$ in the third stage sample). 


\subsubsection{CFA}

Next, CFA was performed for a new dataset $(\mathrm{N}=348)$, and it revealed that the goodness of fit index values were $x^{2} / D F=1.97, x^{2}=192.74, \mathrm{DF}=97, \mathrm{CFI}=.93, \mathrm{NFI}=.90, \mathrm{GFI}=.93$, RMSEA $=.05$ (in the fourth stage sample).

\subsection{The reliability of the TSCRRS}

\subsubsection{Internal reliability}

Cronbach's alpha coefficients of $.80, .85, .76, .63$ (in the third stage sample) and $.80, .85$, $.74, .61$ (in the fourth stage sample) were obtained for the self-expansion, self-contraction, self-pruning, and self-adulteration subscales.

\subsubsection{Parallel tests reliability}

Pearson's correlations among the subscales of the TSCRRS and the RSCS were statistically significant $(p<.01)$ (Table 1).

Table 1. Correlations among the subscales of the TSCRRS and the RSCS

\begin{tabular}{lcccccccc}
\hline Variables & 1 & 2 & 3 & 4 & 5 & 6 & 7 & 8 \\
\hline 1. RSCS self-expansion & 1 & $-.26^{* *}$ & $.45^{* *}$ & .10 & $.70^{* *}$ & -.06 & $.33^{* *}$ & $.13^{*}$ \\
2. RSCS self-contraction & & 1 & $-.12^{*}$ & $.42^{* *}$ & $-.14^{* *}$ & $.53^{* *}$ & -.05 & $.27^{* *}$ \\
3. RSCS self-pruning & & & 1 & $.20^{* *}$ & $.48^{* *}$ & $.11^{*}$ & $.52^{* *}$ & $.32^{* *}$ \\
4. RSCS self-adulteration & & & & 1 & $.28^{* *}$ & $.78^{* *}$ & $.12^{*}$ & $.30^{* *}$ \\
5. TSCRRS self-expansion & & & & & 1 & -.02 & $.38^{* *}$ & $.14^{* *}$ \\
6. TSCRRS self-contraction & & & & & & 1 & .01 & $.42^{* *}$ \\
7. TSCRRS self-pruning & & & & & & & 1 & $.12^{*}$ \\
8. TSCRRS self-adulteration & & & & & & & & 1 \\
\hline $\mathrm{x}$ & 5.08 & 2.07 & 4.31 & 3.07 & 4.51 & 3.02 & 3.98 & 3.57 \\
DF & 1.28 & 1.31 & 1.55 & 1.20 & 1.24 & 1.43 & 1.55 & 1.47 \\
Cronbach's alpha & .82 & .84 & .89 & .77 & .80 & .85 & .74 & .61 \\
\hline$* * p<.01, * p<.05$ & & & & & & & &
\end{tabular}

\subsubsection{Item-total correlations}

To examine the correlations, each item score and overall assessment score of the item-total correlations were calculated. The corrected item-total correlations ranged between .28 and .48 (in the fourth stage sample).

\subsection{The validity and reliability of the IOS Scale}

We only assessed the test-retest reliability and criterion-related validity of the IOS Scale because this scale only contains one item.

\subsubsection{Test-retest reliability}

To examine the test-retest reliability of the IOS Scale, 31 students were re-administered the IOS Scale two weeks after they were first administered it. The results showed that the testretest reliability of the scale was .57 . 


\subsubsection{The criterion-related validity}

To check the correlations between the inclusion of other in the self and the external variables (relationship satisfaction and self-change), the RAS and the RSCS were used. The results revealed that the criterion-related validity of the IOS Scale was satisfactory (Table 2).

Table 2. The criterion-related validity of the IOS Scale

\begin{tabular}{|c|c|c|c|c|c|c|}
\hline Variables & 1 & 2 & 3 & 4 & 5 & 6 \\
\hline 1. RSCS self-expansion & 1 & $-.26 * *$ & $.45^{* *}$ & .10 & $.42^{* *}$ & $.33 * *$ \\
\hline 2. RSCS self-contraction & & 1 & $-.12 *$ & $.42 * *$ & $-.45 * *$ & $-.19^{*}$ \\
\hline 3. RSCS self-pruning & & & 1 & $.20^{* *}$ & $.25^{* *}$ & $.27 * *$ \\
\hline 4. RSCS self-adulteration & & & & 1 & -.11 & .06 \\
\hline 5. Relationship satisfaction & & & & & 1 & $.46^{* *}$ \\
\hline 6. Inclusion of other in the self & & & & & & 1 \\
\hline $\mathrm{X}$ & 5.08 & 2,07 & 4,31 & 3,07 & 5.65 & 5.60 \\
\hline DF & 1.28 & 1.31 & 1.55 & 1.20 & 1.13 & 1.55 \\
\hline Cronbach's alpha & .82 & .84 & .89 & .77 & .89 & - \\
\hline
\end{tabular}

\section{Conclusion}

This study had two aims. One aim was to adapt the IOS Scale into the Turkish cultural context. The second aim was to develop the TSCRRS based on the Two-dimensional Relational Self-change Model. To adapt the IOS Scale, linguistic equivalence was tested at the beginning of the study, and the result showed that the Turkish version of the scale had good linguistic equivalence. After testing for linguistic equivalence, the validity and reliability analyses (only test-retest reliability and criterion-related validity) of the IOS Scale were run. The analyses revealed that the IOS Scale had good validity and reliability. As we expected, there were significant correlations between the IOS Scale and the subscales of the RSCS. At the same time, we created an item-pool to develop the TSCRRS and to measure self-change processes in romantic relationships based on the existing RSCS, the Two-dimensional Model of the Relational Self-change, and a focus group conducted with Turkish college students. After these items were examined by four specialists in the field of close relationships, the authors created the final item-pool. Then, EFA, CFA, parallel-test validity, and criterion-related reliability were performed. The findings demonstrated that the TSCRRS had good validity and reliability. Moreover, as we expected, there were significant correlations between the subscales of TSCRRS and the original RSCS. In conclusion, it is clear that the results regarding the psychometric properties of the TSCRRS and the IOS Scale guarantee that they can be used confidently in couples and marriage counseling research and practices.

Although this study represents a remarkable effort to enrich the close relationships literature, it has several limitations. First, the study sample only consisted of undergraduate students in Turkey. In other words, the present study validated the TSCRRS only in the Turkish cultural context. Although it contains several culture-specific items based on a focus group, in future studies, researchers could validate the TSCRRS in different cultures (i.e., collectivistic and individualistic cultures) in countries around the world and so, they examine the cross-cultural differences on self-change processes. Second, the educational level of the participants was relatively high because the data were collected from an educated sample (university students). In the future, studies could examine these selfchange processes in romantic relationships in low educated samples. 


\section{Acknowledgements}

The authors would like to thank the Scientific and Technological Research Council of Turkey (TUBITAK) 2214/A International Doctoral Research Fellowship Program for supporting this study (and Dr. Duygu Dincer's dissertation) financially.

\section{References}

1. B. A. Mattingly, G. W. Lewandowski, \& K. McIntyre "You make me a better/worse person: A two-dimensional model of relationship self-change." Pers Relationship, 21, 176-190 (2014)

2. K. McIntyre, B.A. Mattingly, \& G. W. Lewandowski When "we" changes "me": The two-dimensional model of relational self-change and relationship outcomes. J Soc Pers Relat, 32(2), 857-878 (2015)

3. A. Aron, \& A. Aron. Love and expansion of self: Understanding attraction and satisfaction (Hemisphere publishing Corporation, New York, 1986)

4. A. Aron, \& E. Aron Self-expansion motivation and including other in the self. In S. Duck (Ed.), Handbook of personal relationships (2nd ed., pp. 251-270). (Wiley, Chichester, UK, 1997)

5. D. Mashek, B. Le, K. Israel, \& A. Aron Wanting less closeness in romantic relationships. Basic Appl SocPsych, 33, 333-345 (2011)

6. A. P. Aron, D. J. Mashek, \& E. A. Aron Closeness a including other in the self. In D.J. Mashek \& A. Aron (Eds.) Handbook of closeness and intimacy (pp. 27-43). (Lawrence Erlbaum Associates, Inc., Publishers, New Jersey, 2008)

7. A. Aron, E. N. Aron, \& D. Smollan Inclusion of other in the self scale and the structure of interpersonal closeness. J Pers Soc Psychol, 63, 596-612 (1992)

8. D. Dincer Factors predicting relationship satisfaction and life satisfaction in romantic relationships: The two-dimensional model of relational self-change (Unpublished dissertation) (Marmara University, Istanbul, Turkey, 2017)

9. D. Dincer, H. Eksi, \& A. Aron Psychometric properties of the Turkish version of the Relational Self-Change Scale. Paper presented at 2nd Eurasian Congress on Positive Psychology, Istanbul, Turkey (2017).

10. S. S. Hendrick A generic measure of relationship satisfaction. J Marriage Fam, 50, 9398 (1988)

11. F. Curun The effects of sexism and sex role orientation on romantic relationship satisfaction (Unpublished thesis). (Middle East Technical University, Ankara, Turkey, 2001) 